\title{
QUADRUPOLE MAGNETS FOR THE 20MEV FFAG, 'EMMA'
}

\author{
B.J.A. Shepherd ${ }^{\#}$, N. Marks, ASTeC, STFC Daresbury Laboratory, Warrington WA4 4AD, UK
}

\section{Abstract}

EMMA is a $20 \mathrm{MeV}$ non-scaling Fixed Field Alternating Gradient accelerator (nsFFAG) proof-ofprinciple prototype, to be built at the Daresbury Laboratory as an accelerator physics experiment to explore the behaviour of such machines. Non-scaling FFAGs have potential applications in charged particle cancer therapy and also for particle physics; however, to date, no such accelerator has been constructed. The magnet designs present major challenges - the lattice is made up of 84 quadrupoles, with different horizontal offsets from the magnet centres in the focusing and defocusing quads. These offsets alone provide the necessary bending fields in the ring. The magnets are also very thin (55mm and $65 \mathrm{~mm}$ yoke lengths) and end field effects therefore dominate. Careful design, followed by prototype construction and measurement, is essential. The magnets have been designed in 3D from the outset, using the CST EM Studio software. The paper will present the results of the design, showing how the magnets have been optimised to improve the integrated good gradient region, and will report on the progress of the prototyping work.

\section{INTRODUCTION}

The Electron Machine for Many Applications (EMMA) will be a non-scaling Fixed Field Alternating Gradient (nsFFAG) accelerator as an add-on to the Energy Recovery Linac Prototype (ERLP) [1] project at Daresbury Laboratory. nsFFAGs could be used to accelerate ions for cancer therapy, as well as being a promising way of delivering rapid acceleration of muons required in a neutrino factory. EMMA is part of the CONFORM project [2], funded within the BASROC initiative, and is a proof-of-principle machine. It will take a $10 \mathrm{MeV}$ beam from the ERLP, accelerate it up to $20 \mathrm{MeV}$ and extract it into a diagnostic beamline.

The EMMA lattice is described fully elsewhere in these proceedings [3]. The main lattice magnets are 84 quadrupoles (42 each of $F$ and D types), which are offset to provide a dipole field, and therefore effectively work as combined function magnets. The magnets will be movable in the horizontal direction to provide independent control of the dipole and quadrupole fields.

\section{MAGNET CHALLENGES}

Due to the small size of the EMMA ring $(16.6 \mathrm{~m}$ circumference) and the large number of magnets, each magnet is very thin - the yoke thicknesses are the same order as the inscribed radii. The field is therefore dominated by end effects, which in conventional storage ring magnets are small corrections.

In an FFAG, the beam moves significantly inside the vacuum chamber as it is ramped in energy. The required horizontal aperture is therefore rather large, and consequently the good field region specified for the magnets is quite demanding.

The size of the ring is such that all the EMMA components (magnets, cavities, injection/extraction magnets, diagnostics and correctors) must fit into an extremely small space. Interaction between the two magnets in a cell must be taken into consideration, as well as fields in the straight sections.

Full 3D modelling using CST EM Studio [4] has been employed from the outset, and the results have been cross-checked with OPERA-3D [5]. A pair of prototype magnets is being built by Tesla Engineering [6], to verify the simulation work.

\section{MAGNET PARAMETERS}

Table 1 shows a list of current magnet parameters. The individual magnet profiles are shown in Figures 1 and 2. The required good field regions are highlighted with a rounded rectangle in each case. Note that the good field region for the $\mathrm{D}$ magnet is entirely offset from the magnetic centre.

Table 1: List of Current Magnet Parameters for EMMA

\begin{tabular}{|l|l|l|l|}
\hline Parameter & F magnet & D magnet & Units \\
\hline Integrated gradient & -0.387 & 0.347 & $\mathrm{~T}$ \\
\hline Inscribed radius & 37 & 53 & $\mathrm{~mm}$ \\
\hline Current & 213.4 & 263.5 & $\mathrm{~A}$ \\
\hline Turns in coil & 11 & 11 & \\
\hline Yoke thickness & 55 & 65 & $\mathrm{~mm}$ \\
\hline Pole width & 73 & 100 & $\mathrm{~mm}$ \\
\hline $\begin{array}{l}\text { Horizontal } \\
\text { movement range }\end{array}$ & $\begin{array}{l}-2.711 \\
+2.604\end{array}$ & $\begin{array}{l}-5.28 \\
+14.535\end{array}$ & $\mathrm{~mm}$ \\
\hline $\begin{array}{l}\text { Offset from } \\
\text { magnetic centre }\end{array}$ & 7.507 & 34.025 & $\mathrm{~mm}$ \\
\hline $\begin{array}{l}\text { Required good field } \\
\text { region }\end{array}$ & $-32 \ldots+16$ & $-56 \ldots-10$ & $\mathrm{~mm}$ \\
\hline
\end{tabular}

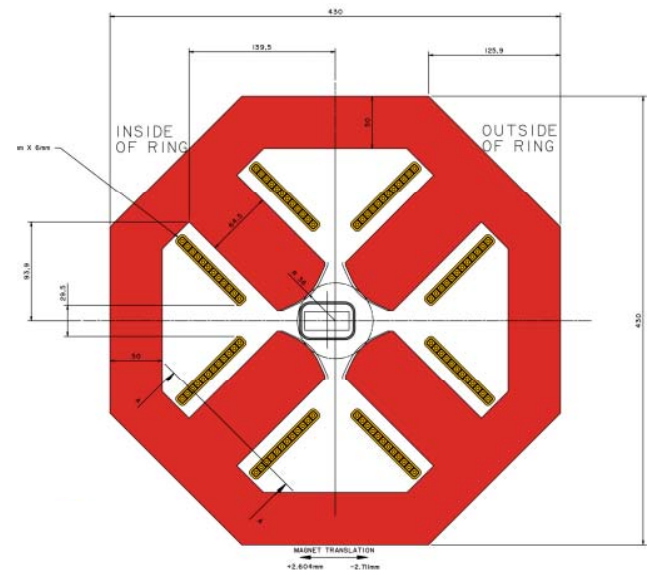

Figure 1: F magnet profile. 


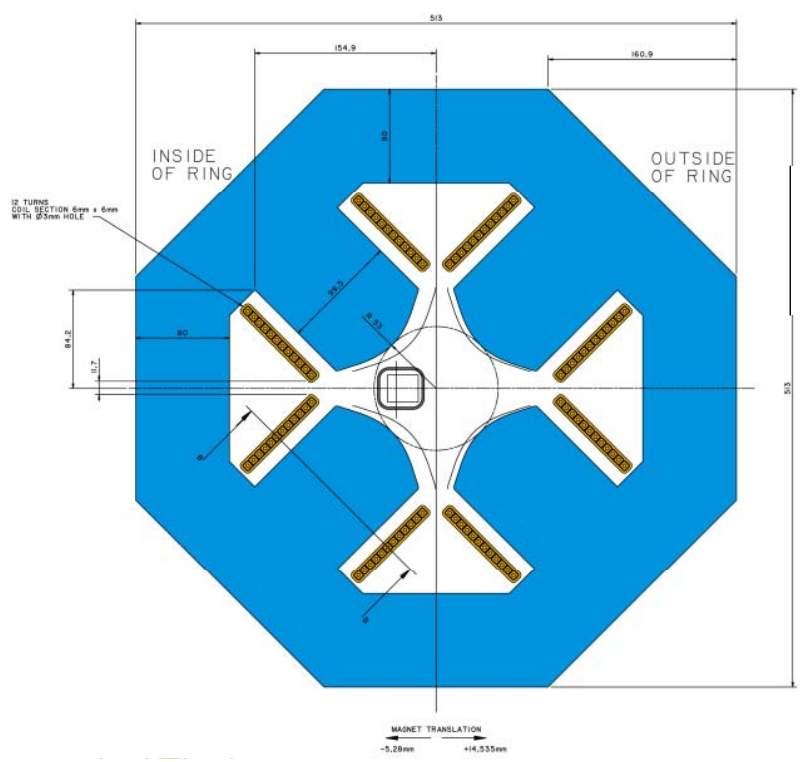

Figure 2: D magnet profile.

MAGNET MODELLING

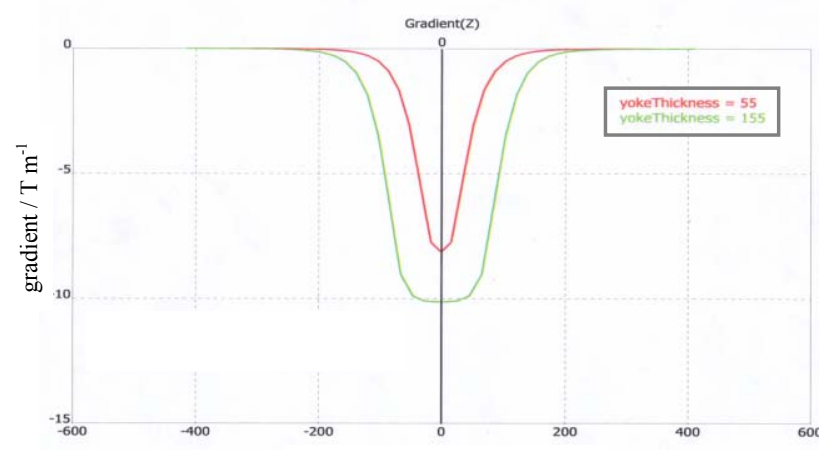

Figure 3: Gradient profile through the F magnet using two different values of yoke thickness. The gradient from the $155 \mathrm{~mm}$-thick magnet reaches a plateau value, but the $55 \mathrm{~mm}$-thick magnet does not.

A 2D model of the $\mathrm{F}$ magnet was produced in OPERA, to find a ballpark figure for the tangent point - the point at which the pole profile goes from a hyperbolic curve to a tangent. 3D modelling suggested that, due to the very short magnet length, the central gradient was not as high as the 2D model. The length would have to be at least doubled to reach the 'plateau' value (Figure 3). This confirms that the field is dominated by end effects. The central gradient is therefore smaller than that expected from the 2D model; however, the integrated gradient is rather larger than naïvely expected (by multiplying the central value by the yoke length).

Interaction between the F and D magnets in a cell was assessed by building three models: two separate models and one with both magnets. The difference (in gradient) between a linear addition of the separate models and the combined model was found to be about $0.25 \mathrm{~T} / \mathrm{m}$ at the centre of the F magnet - about $5 \%$ of the central gradient (Figure 4).

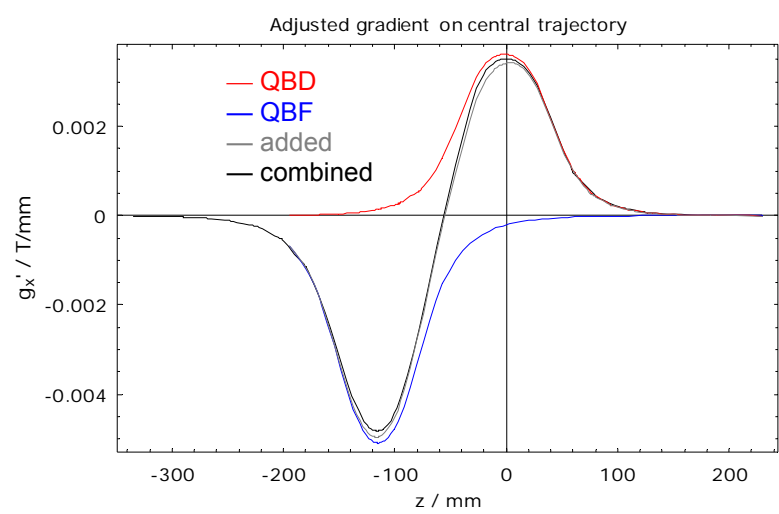

Figure 4: Gradient on the nominal $15 \mathrm{MeV}$ beam path, showing the two individual magnets (red and blue), a linear superposition of these (grey) and a combined model (black).

The injection line design [7] specifies a kicker that will not be affected by magnetic field from the $\mathrm{F}$ and $\mathrm{D}$ magnets. To limit the amount of field leaking into the long straight, field clamps were added to the design. Thin $(5 \mathrm{~mm})$ steel plates with a hole for the vacuum chamber will be added on either side of every F-D pair, providing a return path for the stray magnetic flux and decreasing the field in the long straight to acceptable levels.

Each magnet was optimised separately in EM Studio, with a view to tweaking the combined model at a later stage and providing a field map to use in tracking studies, iterating the design further. The goal was to achieve as large a possible region within which the integrated gradient variation did not exceed $\pm 0.1 \%$. The design goals were specified as $\pm 32 \mathrm{~mm}$ for the $F$ magnet and $\pm 56 \mathrm{~mm}$ for the D magnet. These apertures are defined by the required beam movement as the energy changes, plus the horizontal movement specified for each magnet.

Initially, two variables were used in the simulation tangent point and the size of the chamfer at the pole ends. For a normal (long) storage ring quadrupole, this would give sufficient degrees of freedom - adjusting the tangent point to correct the central field, and then adding a chamfer to correct for end effects. However, in these very short magnets, the end fields dominate the overall field quality, and the gradient map seems to have features which cannot be corrected for using these variables alone. The maximum good field region available using this geometry (including a field clamp) was $\pm 14 \mathrm{~mm}$.

A new approach was tried, changing from the old pole face model that used a hyperbolic section and a tangent section.

\section{'Straight-Line' Pole Geometry}

An arbitrary pole design provides the freedom to adjust the field profile with fewer restraints than that imposed by a 'traditional' quadrupole design. However, it raises the question of how to parametrise the pole. The model initially tried was based on the following steps (see Figure 5 for definition of parameters):

- Begin with a square pole. 
- Remove material from each side of the pole, adjusting the $d_{0}$ point until reaching an optimum.

- Adjust the $d_{l}$ point, halfway between the pole centre and the side.

- Introduce a third $\left(d_{2}\right)$ point halfway between the two previous points, and adjust this.
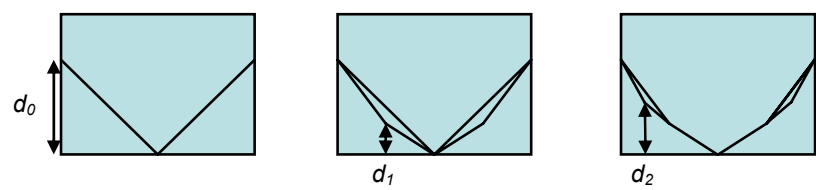

Figure 5: Optimisation of straight-line geometry for an even number of pole tip facets. Points are introduced one at a time, optimising at each step.

This method turns out to be very successful in generating a pole geometry that conforms to the specification. Without field clamps, the good field region was extended to $\pm 32 \mathrm{~mm}$. Adding the field clamp to the model, however, has an adverse effect on the field quality.

The shape of the vacuum window in the field clamp was altered to try to improve the field quality. Various different shapes were tried (Figure 6), but the best was found to be a shape following the outline of the magnet poles. This has the advantage of keeping the quadrupole symmetry, so that field quality in the vertical direction does not require further evaluation.
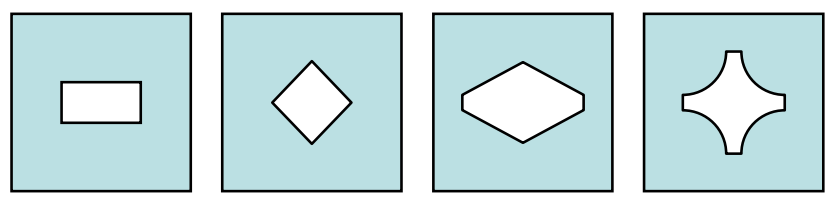

Figure 6: Differing vacuum window shapes in the field clamp: rectangle, diamond, tapered rectangle, and following the pole shape.

A variant of the straight-line pole tip geometry was tried in which an odd number of pole tip faces were used. Designs with three and five faces, using one and two variables respectively, were tried out (Figure 7). The optimisation was done sequentially as above, based on the assumption that the two variables were fairly orthogonal.
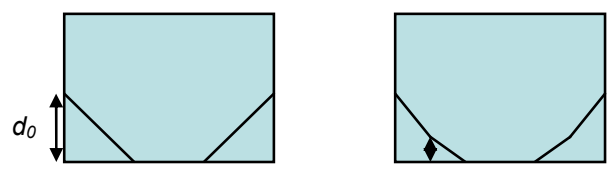

Figure 7: Optimisation of straight-line geometry for an odd number of pole tip facets (three and five).

For the $\mathrm{F}$ magnet, the best result was found for a fiveface geometry with $d_{0}=19.5 \mathrm{~mm}$ and $d_{1}=4.25 \mathrm{~mm}$, resulting in a good field region of $\pm 22.9 \mathrm{~mm}$ (Figure 8). This is somewhat short of the specified value of $32 \mathrm{~mm}$. It may become clearer whether this could be acceptable or not when tracking studies are carried out using real simulated field maps from this study.

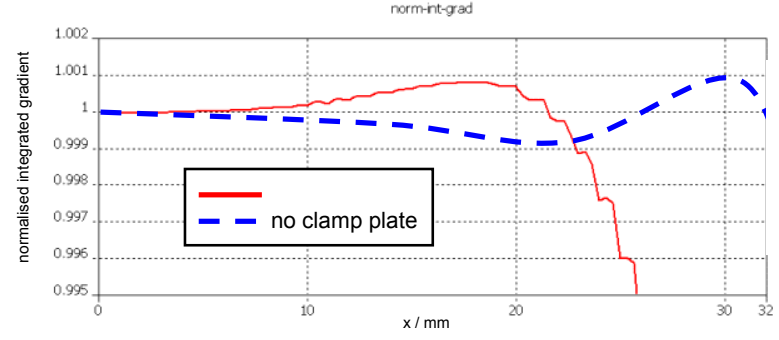

Figure 8: Normalised integrated gradient plot of the optimum configuration for the F magnet. The good field region is $22.9 \mathrm{~mm}$. A plot for a magnet with no clamp plate is also shown.

\section{FURTHER WORK}

Full optimisation of the D magnet is yet to be carried out. When this is completed, a full combined model of the entire cell can be built and used in tracking codes to simulate injection, transport and extraction of the EMMA beam from $10-20 \mathrm{MeV}$. Results from tracking could be used to further tweak the magnets.

Prototypes of these magnets have been ordered, and will be complete by the end of September 2007. Optimisation of the magnet design will continue in parallel with the order - discussions with the magnet manufacturer are ongoing. The quadrupoles will be tested at the dedicated magnet test facility at Daresbury Laboratory to ensure they meet the specifications.

The field clamp was introduced primarily to reduce stray fields in the kicker straights. Once some detailed kicker modelling has been carried out, it may be possible to modify the field clamp geometry, and try to improve the field quality in the quadrupoles.

\section{CONCLUSIONS}

Magnet modelling has been carried out for EMMA's extremely demanding ring magnets. The field is dominated by end effects, and a conventional hyperbola/tangent pole tip geometry does not meet the specification. A novel straight-line geometry produces a larger good field region.

\section{REFERENCES}

[1] S.L. Smith, "The Status of the Daresbury Energy Recovery Linac Prototype", these proceedings.

[2] R.J. Barlow, "The CONFORM Project: Construction of a Nonscaling FFAG and its Applications", these proceedings.

[3] J.S. Berg, "The EMMA Lattice Design", these proceedings.

[4] CST simulation software, www.cst.com.

[5] OPERA magnet modelling software, www.vectorfields.com.

[6] Tesla Engineering, www.tesla.co.uk.

[7] T. Yokoi, "Beam Injection and Extraction in EMMA NS-FFAG Ring", these proceedings. 